\title{
On the "direct" calculation of thermal rate constants. II. The flux-flux autocorrelation function with absorbing potentials, with application to the $\mathrm{O}+\mathrm{HCl} \rightarrow \mathrm{OH}+\mathrm{Cl}$ reaction
}

\author{
Ward $\mathrm{H}$. Thompson and William H. Miller ${ }^{\mathrm{a}}$ \\ Department of Chemistry, University of California, Berkeley, California 94720-1460 \\ and Chemical Sciences Division, Lawrence Berkeley Laboratory, Berkeley, California 94720
}

(Received 20 August 1996; accepted 24 September 1996)

\begin{abstract}
We present a method for obtaining the thermal rate constant directly (i.e., without first solving the state-to-state reactive scattering problem) from the time integral of the flux-flux autocorrelation function, $C_{f f}(t)$. The quantum mechanical trace involved in calculating $C_{f f}(t)$ is efficiently evaluated by taking advantage of the low rank of the Boltzmannized flux operator. The time propagation is carried out with a Hamiltonian which includes imaginary absorbing potentials in the reactant and product exit channels. These potentials eliminate reflection from the edge of the finite basis and ensure that $C_{f f}(t)$ goes to zero at long times. In addition, the basis can then be contracted to represent a smaller area around the interaction region. We present results of this method applied to the $\mathrm{O}+\mathrm{HCl}$ reaction using the $J$-shifting and helicity conserving approximations to include nonzero total angular momentum. The calculated rate constants are compared to experimental and previous theoretical results. Finally, the effect of deuteration (the $\mathrm{O}+\mathrm{DCl}$ reaction) on the rate constant is examined. (C) 1997 American Institute of Physics. [S0021-9606(97)01701-7]
\end{abstract}

\section{INTRODUCTION}

As implied by the title, this article is a continuation of our quest to find the most powerful general procedure for calculating the thermal rate constant of a bimolecular chemical reaction correctly and directly, i.e., one that is in principle exact (given a potential energy surface) and also one that avoids having to solve explicitly the complete state-tostate quantum reactive scattering problem. Our efforts, ${ }^{1,2}$ and those of a number of other workers, ${ }^{3-11}$ are based on the formally exact expression for the rate constant as the time integral of the flux-flux autocorrelation function ${ }^{12,13}$

$$
k(T)=Q_{r}(T)^{-1} \int_{0}^{\infty} d t C_{f f}(t),
$$

where

$$
C_{f f}(t)=\operatorname{tr}\left[\hat{F} e^{i \hat{H} t_{c}^{*} / \hbar} \hat{F} e^{-i \hat{H}_{c} / \hbar}\right],
$$

or, formally equivalently, as the long time limit of the fluxside correlation function ${ }^{13,14}$

$$
k(T)=Q_{r}(T)^{-1} \lim _{t \rightarrow \infty} C_{f s}(t),
$$

where

$$
C_{f s}(t)=\left[\hat{h} e^{i \hat{H} t_{c}^{*} / \hbar} \hat{F} e^{-i \hat{H} t_{c} / \hbar}\right] .
$$

In Eqs. (1.1) and (1.2) $\hat{H}$ is the Hamiltonian operator of the molecular system, $\hat{F}$ is the symmetrized flux operator (defined with respect to some dividing surface through the interaction region), $\hat{h}$ is a step function that is 1 or 0 on the reactant or product side of the dividing surface, respectively,

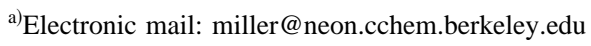

and $t_{c}=t-i \hbar \beta / 2$, where $\beta$ is related to temperature in the usual way, $\beta=\left(k_{B} T\right)^{-1} ; Q_{r}(T)$ is the reactant partition function per unit volume.

Our most recent work ${ }^{2}$ (hereafter referred to as Part I) on this topic utilized Eq. (1.2) and emphasized that, by evaluating $C_{f s}(t)$ at a sufficiently large but finite time $t$, one could avoid having to introduce an absorbing potential to enforce outgoing wave boundary conditions. Though this is indeed true, our present feeling is that the price paid for dispensing with the absorbing potential is too great, i.e., the size of the $L^{2}$ basis (or grid) must be enlarged too much to make this the optimum procedure. This becomes particularly evident when dealing with multidimensional systems where the flux exiting the interaction region has a broad distribution of translational energies: the flux exiting most rapidly will hit the edge of the grid (and undergo unphysical reflection) before the more slowly exiting flux has escaped the interaction region.

The primary change in strategy from Part I, therefore, is to utilize absorbing potentials to make the $L^{2}$ basis (e.g., a discrete variable representation ${ }^{15-17}$ ) as small as possible-in essentially the same way they have been used before ${ }^{18-24}$ - and to put up with the minor nuisance of having to perform test calculations to insure that the results are insensitive to them.

Once an absorbing potential is added to the Hamiltonian,

$$
\hat{H} \rightarrow \hat{H}-i \hat{\epsilon},
$$

one can no longer use Eq. (1.2) to obtain the rate constant (because then $C_{f s}(t) \rightarrow 0$ as $t \rightarrow \infty$ ). Eq. (1.1) is still valid, however, because only the long time behavior is influenced by the absorbing potentials, and the exact $C_{f f}(t) \rightarrow 0$ as $t \rightarrow \infty$ and is thus unaffected by them. Section II describes the general procedure for evaluating $C_{f f}(t)$, where again a key feature is to exploit the low rank of an effective flux operator 
to minimize the number of operations of the time evolution operator that is required. Of previous work, the methodology presented in Section II is most akin to that of Park and Light $^{4(\mathrm{~b})}$ and Brown and Light, ${ }^{5}$ though there are key differences, primarily in the basis used to evaluate the trace and in the use of iterative methods for the Boltzmann operator and the time evolution operator.

Section III then describes the specifics of the methodology with regard to a nontrivial application, a full three dimensional calculation of the rate constant for the $\mathrm{O}+\mathrm{HCl} \rightarrow \mathrm{OH}+\mathrm{Cl}$ reaction. Section IV discusses the results of these calculations including the $H \rightarrow D$ isotope effect, over the temperature range $T=200-700 \mathrm{~K}$.

\section{EVALUATION OF THE FLUX-FLUX AUTOCORRELATION FUNCTION}

A crucial element in the efficient calculation of the flux correlation function of Eq. (1.1b) is to exploit the low rank of some appropriate operator. (The operand of the trace in Eq. (1.1b) is itself of low rank, but it is not possible to exploit this as was done in Part I for $C_{f s}(t)$, because here we need $C_{f f}(t)$ for a range of $t$ while in Part I we needed $C_{f s}(t)$ only for one (large) value of $t$.) For this purpose it is useful to "unbundle" the Boltzmann and (real) time evolution operators in Eq. (1.1b) and write the correlation function in the following equivalent form

$$
C_{f f}(t)=\operatorname{tr}\left[\hat{F}(\beta) e^{i \hat{H} t / \hbar} \hat{F} e^{-i \hat{H} t / \hbar}\right],
$$

where $\hat{F}(\beta)$ is the Boltzmannized flux operator (similar to the one defined by Park and Light ${ }^{4(b)}$ ),

$$
\hat{F}(\beta)=e^{-\beta \hat{H} / 2} \hat{F} e^{-\beta \hat{H} / 2} \text {. }
$$

Though the flux operator $\hat{F}$ itself is not of low rank for a multidimensional system, the Boltzmannized flux operator of Eq. (2.1b) is effectively so. To see this, let us suppose for illustrative purposes that the reaction coordinate (the one degree of freedom for motion normal to the dividing surface) were separable from all the degrees of freedom for motion on the dividing surface (the "activated complex"'); $\hat{F}(\beta)$ would then be given by

$$
\hat{F}_{\text {sep }}(\beta)=\hat{F}_{1 D} e^{-\beta \hat{H}^{\ddagger}}=\hat{F}_{1 D} \sum_{n^{\ddagger}}\left|n^{\ddagger}\right\rangle\left\langle n^{\ddagger}\right| e^{-\beta E_{n^{\ddagger}}},
$$

where $\hat{F}_{1 D}$ is the one dimensional (1D) flux operator (of rank $2)^{4,25}$ and $\hat{H}^{\ddagger}$ the Hamiltonian for the activated complex, with eigenfunctions $\left|n^{\ddagger}\right\rangle$ and eigenvalues $E_{n}$. The effective rank of $\hat{F}_{\text {sep }}(\beta)$ is thus twice the number of states of the activated complex that are significantly populated thermally at temperature $T$. One expects the rank of the true Boltzmannized flux operator to be similar.

The first step of the calculation is therefore to find the eigenfunctions of the Boltzmannized flux operator, $\hat{F}(\beta)$ of Eq. (2.1b), that have the largest (in absolute value) eigenvalues, and the Lanczos algorithm ${ }^{26}$ is ideal for this purpose. Starting with an initial random unit vector $\mathbf{v}_{0}$ (in a finite basis set representation), the sequence of Krylov unit vectors $\left\{\mathbf{v}_{n}\right\}$ is generated by

$$
\begin{aligned}
& \mathbf{v}_{1} \propto \mathbf{F}(\beta) \cdot \mathbf{v}_{0}+\mathrm{SO}, \\
& \mathbf{v}_{2} \propto \mathbf{F}(\beta) \cdot \mathbf{v}_{1}+\mathrm{SO},
\end{aligned}
$$

etc., where "SO" implies a Schmidt orthogonalization to previous vectors. The matrix of $\mathbf{F}(\beta)$ in this orthonormal basis,

$$
\mathbf{F}_{k, k^{\prime}}(\beta)=\mathbf{v}_{k}^{\dagger} \cdot \mathbf{F}(\beta) \cdot \mathbf{v}_{k^{\prime}},
$$

is generated automatically by this procedure. Each Lanczos iteration, i.e., each new Krylov vector generated via Eq. (2.3), requires one action of $\mathbf{F}(\beta)$ onto a vector, and the nontrivial aspect of this is the action of the Boltzmann operator $e^{-\beta \mathbf{H} / 2}$ onto a vector, which is accomplished by the split-operator algorithm described in Section III.

Diagonalization of this (relatively small) matrix $\mathbf{F}_{k, k^{\prime}}(\beta)$ produces the eigenvectors $\left\{\mathbf{u}_{n}\right\}$ with the largest (in absolute value) eigenvalues $\left\{f_{n}\right\}$, so that $\mathbf{F}(\beta)$ is then represented as

$$
\mathbf{F}(\beta)=\sum_{n} f_{n} \cdot \mathbf{u}_{n} \mathbf{u}_{n}^{\dagger}
$$

and the trace for the flux correlation function in Eq. (2.1a) is readily evaluated to give

$$
C_{f f}(t)=\sum_{n} f_{n} \mathbf{u}_{n}^{\dagger}(t) \cdot \mathbf{F} \cdot \mathbf{u}_{n}(t)
$$

where $\mathbf{u}_{n}(t)$ is the time evolved eigenstate of $\mathbf{F}(\beta)$,

$$
\mathbf{u}_{n}(t)=e^{-i \mathbf{H} t / \hbar} \cdot \mathbf{u}_{n} .
$$

This (real) time evolution is also carried out by the splitoperator algorithm, which is the method of choice since it produces, at no additional computational effort, the time evolved state-and thus the flux correlation function via Eq. (2.6) — at all intermediate times necessary to perform the integral of $C_{f f}(t)$.

Also, since the split-operator algorithm generates the time evolution sequentially from one time step to the next, the time integral of $C_{f f}(t)$ can be evaluated simultaneously while doing the time evolution, thereby alleviating the need to store the vectors $\mathbf{u}_{n}(t)$ as a function of $t$. Specifically, if the time evolution is carried out in time increments $\Delta t$, then the time integral of the flux correlation function on this grid of time values $\left(t_{l}=l \Delta t, l=0,1, \ldots,\right)$ gives

$$
k(T)=Q_{r}(T)^{-1} \sum_{l=0} w_{l} C_{f f}\left(t_{l}\right),
$$

where $\left\{w_{l}\right\}$ are the weights for the numerical time integration (e.g., trapezoid rule, or Simpson's rule, etc.). With Eq. (2.6) this becomes

$$
k(T)=Q_{r}(T)^{-1} \sum_{n} f_{n} \sum_{l=0} w_{l} \mathbf{u}_{n}^{\dagger}\left(t_{l}\right) \cdot \mathbf{F} \cdot \mathbf{u}_{n}\left(t_{l}\right) .
$$

Since the split-operator algorithm (see Section IIID) generates the time evolution by the iterative process

$$
\mathbf{u}_{n}\left(t_{l+1}\right)=e^{-i \mathbf{H} \Delta t / \hbar} \cdot \mathbf{u}_{n}\left(t_{l}\right),
$$




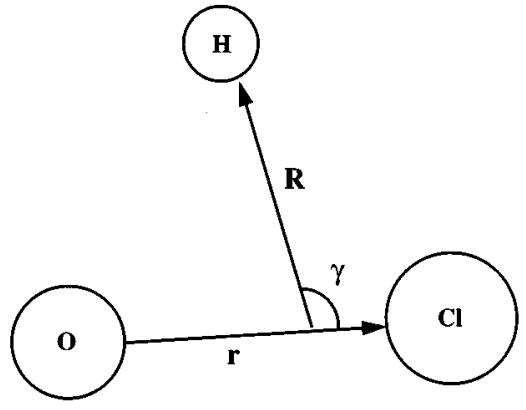

FIG. 1. The Jacobi coordinates of the $\mathrm{H}+\mathrm{OCl}$ arrangement.

after $\mathbf{u}_{n}\left(t_{l}\right)$ is used in Eq. (2.9) to obtain its contribution to the rate and in Eq. (2.10) to produce $\mathbf{u}_{n}(t)$ at the next time step $t_{l+1}=(l+1) \Delta t$, it can be discarded.

To summarize the overall procedure, absorbing potentials are used to keep the grid (or other $L^{2}$ representation) as localized about the interaction region as possible. A Lanczos calculation is first carried out to obtain all the eigenvectors of the Boltzmannized flux operator which have sufficiently large (in absolute value) eigenvalues to contribute to the rate; this is approximately twice the number of states of the activated complex that contribute to the rate. Each of these eigenvectors is time evolved by the split-operator algorithm (which is also used to generate the operator $e^{-\beta \hat{H} / 2}$ in $\hat{F}(\beta))$ and the time integral of $C_{f f}(t)$ evaluated simultaneously.

Section III gives more specifics of the methodology with regard to the $\mathrm{O}+\mathrm{HCl}$ reaction. The significant differences from the earlier work of Light and co-workers ${ }^{4,5}$ are the use of the eigenstates of $\hat{F}(\beta)$ as the basis to evaluate the trace (and the Lanczos method to find them) and an iterative method (split operator) to carry out the real (and imaginary) time evolution.

\section{DETAILS OF CALCULATION}

\section{A. Coordinate system and $J=0$ Hamiltonian}

We have chosen to use the Jacobi coordinates of the $\mathrm{H}+\mathrm{OCl}$ arrangement as shown in Figure 1: $r$ is the $\mathrm{O}-\mathrm{Cl}$ bond distance, $R$ the distance from $\mathrm{H}$ to the center-of-mass of $\mathrm{O}-\mathrm{Cl}$, and $\gamma$ the angle between $\mathbf{r}$ and $\mathbf{R}$. These coordinates describe the interaction region well, and they provide a framework for approximate angular momentum decoupling schemes (such as the helicity conserving approximation or the $J$-shifting approximation ${ }^{27}$ ) since to a good approximation one expects the projection of the total angular momentum along the $\mathrm{O}-\mathrm{Cl}$ axis to be conserved (because the $\mathrm{H}$ atom is so light compared to $\mathrm{O}$ and $\mathrm{Cl}$ ). by

The $J=0$ Hamiltonian in this coordinate system is given

$$
\begin{aligned}
\hat{H}= & -\frac{\hbar^{2}}{2 \mu_{R}} \frac{\partial^{2}}{\partial R^{2}}-\frac{\hbar^{2}}{2 \mu_{r}} \frac{\partial^{2}}{\partial r^{2}}+\left(\frac{1}{2 \mu_{R} R^{2}}+\frac{1}{2 \mu_{r} r^{2}}\right) \hat{\ell}^{2} \\
& +\hat{V}(R, r, \gamma),
\end{aligned}
$$

where $\hat{\ell}^{2}$ is the orbital angular momentum operator associated with the motion of $\mathrm{H}$ about the center of mass of $\mathrm{O}-\mathrm{Cl}$.
$\mu_{R}$ and $\mu_{r}$ are the reduced masses associated with the coordinates $R$ and $r$, respectively.

\section{B. Nonzero total angular momentum}

We have included the effects of nonzero total angular momentum by means of two approximate methods. In the $J$-shifting approximation, ${ }^{27}$ it is assumed that rotational motion and internal motion are separable, so that the rotational energy $E_{J K}$ simply adds to the $J=0$ Hamiltonian of Eq. (3.1), and furthermore, $E_{J K}$ is usually approximated by using rotation constants corresponding to the transition state geometry of the potential energy surface. Following Koizumi et al. ${ }^{28}$ the transition state geometry is assumed to be a symmetric top and the rotational energy levels are given by

$$
E_{J K}=\frac{J(J+1) \hbar^{2}}{2 I_{B}}+\left(\frac{1}{2 I_{A}}-\frac{1}{2 I_{B}}\right) K^{2} \hbar^{2},
$$

where the moments of inertia $I_{B}$ and $I_{A}$ are effectively that of $\mathrm{O}-\mathrm{Cl}$ and of $\mathrm{H}$ about the $\mathrm{O}-\mathrm{Cl}$ axis, respectively. The values used by Koizumi et al. are $I_{B}=4.16 \times 10^{5}$ a.u. and $I_{A}=1.70 \times 10^{3}$ a.u. The total rate constant can then be obtained by a single calculation for $J=0$ as

$$
\begin{aligned}
& k(T)=k_{J=0}(T) Q_{J S}(T), \\
& Q_{J S}(T)=\sum_{J=0}(2 J+1) \sum_{K=-J}^{J} e^{-\beta E_{J K},}
\end{aligned}
$$

where $Q_{J S}(T)$ is the rotational partition function.

The centrifugal sudden, or helicity conserving approximation (HCA), is a more sophisticated approximation in which the Coriolis coupling terms in the body-fixed representation of the Hamiltonian are neglected. ${ }^{29}$ A difference in our present treatment from the usual helicity conserving approximation is that we have chosen the diatom vector $\mathbf{r}$ (the $\mathrm{O}-\mathrm{Cl}$ vector) as the body-fixed quantization axis-because the projection of total angular momentum onto it is most nearly conserved-rather than the atom-diatom vector $\mathbf{R}$ as is usually done. ${ }^{30}$ This results in the following term,

$$
\hat{H}_{\mathrm{HCA}}=\frac{\left[J(J+1)-2 K^{2}\right] \hbar^{2}}{2 \mu_{r} r^{2}}, \quad|K| \leqslant \min (J, \ell),
$$

being added to the $J=0$ Hamiltonian of Eq. (3.1). Within this approximation $J$ and $K$ are conserved quantum numbers and appear simply as parameters in the Hamiltonian. One calculates the rate via Eq. (2.9) for each $J$ and $K$, and then the total rate constant is given by

$$
k(T)=\sum_{J=0}(2 J+1) \sum_{K=-J}^{J} k_{J K}(T) .
$$

The helicity conserving approximation is thus more expensive to apply than the $J$-shifting approximation because the latter requires only the $J=0$ calculation (cf. Eq. (3.3)) whereas the former requires a separate calculation-each of which is essentially the effort of the $J=0$ calculation-for each value of $J$ and $K$. In practice, though, things are greatly simplified because the dependence of $k_{J K}$ on $J$ and $K$ is very simple. For example, if the $J$-shifting approximation were accurate, then Eq. (3.3) shows the $J$ and $K$ dependence is

$$
\ln k_{J K}(T)=\text { constant }-\alpha J(J+1)-\eta K^{2} .
$$


It will be seen in Section III D that Eq. (3.6) is not quantitatively correct, but nevertheless one needs to carry out calculations for only a few values of $J$ and $K$ and interpolate between them in order to evaluate the sum in Eq. (3.5).

\section{Basis set}

We have chosen to use a discrete variable representation (DVR) basis. ${ }^{15-17}$ This has the advantages that the Hamiltonian is sparse in a multidimensional case and no integrals over the potential need to be evaluated. Specifically, we have used the radial sinc-function DVR developed by Colbert and Miller ${ }^{17}$ for the $r$ and $R$ coordinates and a Gauss-Legendre DVR for the $\gamma$ coordinate. In the case where the HC approximation is used, an associated Legendre DVR is used since the projection quantum number is nonzero. ${ }^{31-34}$

The basis set is defined by the parameters $N_{B}, N_{\gamma}$, $V_{c u t}$, and $R_{\max }$. The radial sinc-function DVR has evenly spaced points with the grid spacing $\Delta x$ determined by the maximum kinetic energy in the problem. The grid constant, $N_{B}$, determines the number of points per thermal de Broglie wavelength for the $R$ and $r$ coordinates:

$$
\Delta x=\frac{2 \pi}{N_{B}}\left(\frac{2 \mu k_{B} T}{\hbar^{2}}\right)^{-1 / 2} .
$$

For the present applications we have found $N_{B}=11-13$ to be sufficiently large. $N_{\gamma}$ is the number of Gauss-Legendre DVR points used for the $\gamma$ coordinate (usually, $N_{\gamma} \simeq 30$ ). A "raw" grid is laid down in the Jacobi coordinates of the $\mathrm{H}+\mathrm{OCl}$ arrangement and truncated by an energy cuttoff: if the potential energy at a DVR point is greater than $V_{\text {cut }}$, that point is discarded. The grid is also truncated in the asymptotic reactant, $\mathrm{O}+\mathrm{HCl}$, valley if the translational Jacobi coordinate is greater than $R_{\max }$. It is similarly truncated in the $\mathrm{OH}+\mathrm{Cl}$ and $\mathrm{H}+\mathrm{OCl}$ valleys using the translational Jacobi coordinates of those arrangements.

\section{Time propagation}

The Hamiltonian in Eq. (3.1) can be written as

$$
\hat{H}=\hat{T}_{R}+\hat{T}_{r}+\hat{T}_{\gamma}+\hat{V},
$$

including the total angular momentum centrifugal potential in the term $\hat{V}$. Note that the radial kinetic energy operators, $\hat{T}_{R}$ and $\hat{T}_{r}$, do not commute with the angular kinetic energy operator $\hat{T}_{\gamma}$ or the potential operator $\hat{V}$. Following Zhang and Zhang ${ }^{35}$ we first form a split-operator propagator by dividing the Hamiltonian into the radial kinetic energy terms and the angular kinetic energy plus the potential. Noting that $\hat{T}_{R}$ and $\hat{T}_{r}$ commute with each other, this gives

$$
\begin{aligned}
e^{-i(\hat{H}-i \hat{\epsilon}) \Delta t / \hbar} \simeq & e^{-i \hat{T}_{R} \Delta t / 2 \hbar} e^{-i \hat{T}_{r} \Delta t / 2 \hbar} \\
& \times e^{-i\left(\hat{T}_{\gamma}+\hat{V}-i \hat{\epsilon}\right) \Delta t / \hbar} e^{-i \hat{T}_{r} \Delta t / 2 \hbar} e^{-i \hat{T}_{R} \Delta t / 2 \hbar} .
\end{aligned}
$$

Since $\hat{T}_{\gamma}$ and $\hat{V}$ also do not commute, another split-operator propagator is formed for the central term:

$$
e^{-i\left(\hat{T}_{\gamma}+\hat{V}-i \hat{\epsilon}\right) \Delta t / \hbar} \simeq e^{-i \hat{T}_{\gamma} \Delta t / 2 \hbar} e^{-i(\hat{V}-i \hat{\epsilon}) \Delta t / \hbar} e^{-i \hat{T}_{\gamma} \Delta t / 2 \hbar}
$$

Then the final form of the propagator in terms of the individual $1 \mathrm{D}$ propagators is given by

$$
\begin{aligned}
e^{-i(\hat{H}-i \hat{\epsilon}) \Delta t / \hbar} \simeq & e^{-i \hat{T}_{R} \Delta t / 2 \hbar} e^{-i \hat{T}_{r} \Delta t / 2 \hbar} e^{-i \hat{T}_{\gamma} \Delta t / 2 \hbar} \\
& \times e^{-i(\hat{V}-i \hat{\epsilon}) \Delta t / \hbar} e^{-i \hat{T}_{\gamma} \Delta t / 2 \hbar} e^{-i \hat{T}_{r} \Delta t / 2 \hbar} e^{-i \hat{T}_{R} \Delta t / 2 \hbar} .
\end{aligned}
$$

The 1D propagators are applied sequentially using a sparse matrix multiplication scheme. Since only the 1D kinetic energy matrices and the values of the potential need to be stored, the memory requirements are quite low: usually less than $10 \mathrm{MB}$ of core memory and never more than $30 \mathrm{MB}$ even for the largest basis of about 19000 DVR grid points. The optimum time step is determined by calculating the rate constant for successively smaller $\Delta t$ until the result does not change. The Boltzmann operator $e^{-\beta \hat{H} / 2}$ is applied using the same method but in imaginary time, $\Delta t \rightarrow-i \hbar \Delta \beta / 2$ (and without the absorbing potentials).

For the radial coordinates an analytical form for the free particle propagator in the sinc-function DVR can be found in terms of error functions. In addition, in the DVR the potential energy propagator is approximated as a diagonal matrix with the diagonal elements equal to the exponential of the potential evaluated at the specified DVR point. The angular kinetic energy propagator in the Gauss-Legendre DVR is applied to a vector by transforming to the finite basis representation of Legendre polynomials where $\hat{T}_{\gamma}$ (and thus $\left.e^{-i \hat{T}_{\gamma} \Delta t / 2 \hbar}\right)$ is diagonal. The diagonal propagator operates and then the resulting vector is transformed back to the DVR.

\section{E. Dividing surface and flux operator}

We note that there exist multiple expressions for the flux operator that do not possess identical numerical properties in an $L^{2}$ basis representation. We have chose to express the flux operator as

$$
\hat{F}=\frac{i}{\hbar}[\hat{H}, h(s(\mathbf{q}))],
$$

because it is more straightforwardly generalized to higher dimensions and is easily applied with a sparse matrix multiply routine. It is especially more convenient than the differential form when the dividing surface-defined by the equation $s(\mathbf{q})$-is expressed in terms of coordinates other than those used to represent the Hamiltonian. In the DVR, the matrix elements of the flux operator are easily evaluated as

$$
\mathbf{F}_{j, j^{\prime}}=\frac{i}{\hbar} \mathbf{T}_{j, j^{\prime}}\left[h\left(s_{j^{\prime}}\right)-h\left(s_{j}\right)\right],
$$

where $h\left(s_{j}\right)$ is the step function evaluated at the $j$ th DVR point, and $\mathbf{T}_{j, j^{\prime}}$ is the kinetic energy matrix. The dividing surface used in this study is defined by $r_{\mathrm{OH}}-r_{\mathrm{HCl}}+0.29=0$ (with all distances in atomic units). 


\section{F. Absorbing potential}

The absorbing potential is taken to be a function of the translational Jacobi coordinate in each arrangement, $\epsilon_{\tau}=\epsilon_{\tau}\left(R_{\tau}\right)$. There are several satisfactory choices for the functional form of the absorbing potential. However, any choice must turn on slowly enough not to cause reflection, yet be strong enough to absorb all outgoing flux. We have found the quartic potential to work well,

$$
\epsilon_{\tau}\left(R_{\tau}\right)=\lambda\left(\frac{R_{\tau}-R_{0, \tau}}{R_{\max , \tau}-R_{0, \tau}}\right)^{4},
$$

where $\tau$ is the arrangement index. $R_{0, \tau}$ and $R_{\max , \tau}$ are the starting and ending points of the absorbing potential in the $\tau$ arrangement. $\lambda$ is a strength parameter representing the maximum value of the absorbing potential, generally it is taken to be about $1 \mathrm{eV}$. The beginning of the absorbing strip is chosen such that the imaginary potential has significant value only where the interaction potential is small.

\section{RESULTS AND DISCUSSION}

We have calculated the thermal rate constants for the $\mathrm{O}+\mathrm{HCl}$ reaction on the Koizumi, Schatz, and Gordon $(\mathrm{KSG})^{28}$ potential energy surface which is an analytical fit to $a b$ initio calculations, ${ }^{28,36}$ but with the barrier height scaled down from 18.8 to $8.5 \mathrm{kcal} / \mathrm{mole}$ to match the experimental rate $^{37}$ at $T=295 \mathrm{~K}$. This reaction provides a rigid test of a method for calculating thermal rate constants. The heavy masses involved demand a large basis and the heavy-lightheavy nature of the reaction requires that the correlation function be calculated for long times.

There have been numerous experimental measurements of the thermal rate constant for the $\mathrm{O}+\mathrm{HCl}$ reaction, ${ }^{37-42}$ in addition to several theoretical studies. ${ }^{28,38,43,44}$ Brown and Smith $^{38}$ and Persky and Broida ${ }^{43}$ carried out quasiclassical trajectory calculations on semi-empirical London-EyringPolanyi-Sato (LEPS) surfaces. These surfaces all featured a collinear transition state geometry. However, ab initio calculations on the system indicate that the transition state is significantly bent with an $\mathrm{O}-\mathrm{H}-\mathrm{Cl}$ angle of about $135^{\circ} .28,36,45$ The KSG potential energy surface, with a bent transition state, was originally used by Koizumi et al. to calculate total and state-selected thermal rate constants. These calculations were carried out by integrating coupled channel equations in hyperspherical coordinates to obtain the state-to-state reaction probabilities that were then used to obtain the rate constants (with a $J$-shifting approximation). Moribayashi and Nakamura have also carried out quantum reactive scattering calculations on the KSG surface (as well as a LEPS surface of Persky and Broida) by integrating coupled channel equations in hyperspherical coordinates. ${ }^{44}$ They obtained stateselected and cumulative reaction probabilities as well as state-selected (but not total) rate constants. In addition they examined different approximations for including the effects of nonzero total angular momentum.

Recently, $a b$ initio calculations were carried out and a potential energy surface obtained by Ramachandran, Senekowitsch, and Wyatt with a barrier height of 17.8

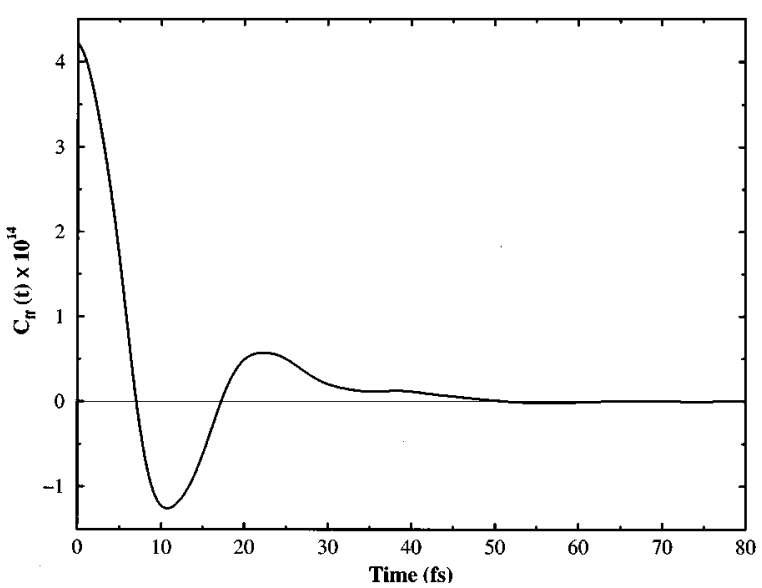

FIG. 2. The flux-flux autocorrelation function for the $\mathrm{O}+\mathrm{HCl}$ reaction at $T=300 \mathrm{~K}$. The units of the correlation function are (atomic units of time) ${ }^{-2}$.

$\mathrm{kcal} / \mathrm{mole}^{45}$ This is in reasonable agreement with the ab initio barrier height of $18.8 \mathrm{kcal} / \mathrm{mole}$ used in the KSG surface before scaling. Their best $a b$ initio estimate of the barrier height is below $11 \mathrm{kcal} / \mathrm{mole}^{46}$ compared to $11.9 \mathrm{kcal} / \mathrm{mole}$ obtained by Gordon et al. ${ }^{36}$ Rate constants have not yet been computed on this surface.

The KSG potential energy surface has a bent transition state geometry with an $\mathrm{O}-\mathrm{H}-\mathrm{Cl}$ angle of $133.4^{\circ}$ and a barrier height of $8.5 \mathrm{kcal} / \mathrm{mole}^{28}$ The $\mathrm{O}+\mathrm{HCl} \rightarrow \mathrm{OH}+\mathrm{Cl}$ reaction is endothermic. In contrast, the $\mathrm{H}+\mathrm{OCl}$ asymptotic arrangement is $\sim 40 \mathrm{kcal} / \mathrm{mole}$ higher in energy than $\mathrm{O}+\mathrm{HCl}$ and is therefore not a relevant product channel. Thus we refer to $\mathrm{OH}+\mathrm{Cl}$ as "products" without ambiguity.

There is an excited $\left({ }^{3} A^{\prime}\right)$ electronic state surface which is degenerate at linear geometries with the $\left({ }^{3} A^{\prime \prime}\right)$ ground state. The details of this surface are not fully known and it is not included in the scattering calculations here. However, following Koizumi et al. ${ }^{28}$ the rate constants presented here have been multiplied by the factor

$$
f(T)=3 /\left(5+3 e^{-228 / T}+e^{-326 / T}\right)
$$

to approximately account for collisions that end up on the excited state as opposed to the ground state surface.

Figure 2 shows the $J=0$ flux-flux autocorrelation function for the $\mathrm{O}+\mathrm{HCl}$ reaction at $T=300 \mathrm{~K}$. At very short times the correlation function decays rapidly and goes through zero around $7 \mathrm{fs}$, corresponding to an initial passage of flux across the dividing surface towards products. This is followed by a negative lobe indicating flux returning across the dividing surface from products to reactants. It is expected that a heavy-light-heavy system such as $\mathrm{O}+\mathrm{HCl}$ should exhibit significant recrossing of the transition state. In a classical picture the $\mathrm{H}$ atom in the region of the transition state is trapped between the massive $\mathrm{O}$ and $\mathrm{Cl}$ and bounces back and forth between these two collision partners. This is manifested in the oscillations in the correlation function-the negative lobe is immediately followed by a positive one representing a second passage of flux towards products. (Of course the oscillations observed depend on the position of the flux di- 


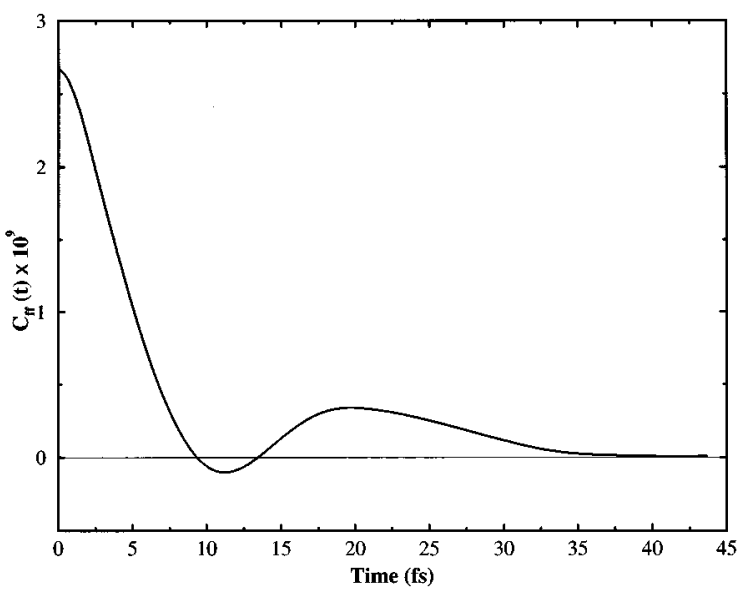

FIG. 3. The flux-flux autocorrelation function for the $\mathrm{O}+\mathrm{HCl}$ reaction at $T=700 \mathrm{~K}$.

viding surface that was not optimized here for minimum recrossing.) The correlation function eventually dies to zero around $50 \mathrm{fs}$. This is twice the time that would be expected in the case of a direct reaction (which would be $\sim \hbar \beta \simeq 25$ fs). ${ }^{13}$

Figure 3 shows the $J=0$ flux-flux autocorrelation function for the $\mathrm{O}+\mathrm{HCl}$ reaction at $T=700 \mathrm{~K}$. This correlation function is similar to that shown in Fig. 2 for $T=300 \mathrm{~K}$. There is a rapid initial decay to zero followed by a negative lobe and then a postive lobe. At this temperature the correlation function decays to zero in approximately $35 \mathrm{fs}$ (cf. $\hbar \beta \simeq 11 \mathrm{fs}$ ). However, in this case the negative lobe is much smaller (relative to the initial value of the correlation function) than for the lower temperature, while the following positive lobe is about the same magnitude. However the smaller oscillations observed at $T=300 \mathrm{~K}$ are not present here. While in a variational transition state theory picture the optimum dividing surface for minimizing recrossing may change with temperature, we have used the same dividing surface at all temperatures.

Figure 4 shows the $(J$-shifted) rate constant versus the number of Lanczos iterations for $T=400 \mathrm{~K}$. Recall that each Lanczos iteration corresponds to an eigenvalue (and eigenvector) of the Boltzmannized flux operator that is included in the calculation of the rate. At this temperature the rate converges with around 20 iterations implying that there are 9 or 10 thermally accessible states of the activated complex contributing to the reaction rate. With the heavy masses of the oxygen and chlorine it is expected there will be more states accesible at a given temperature than for a reaction like $\mathrm{H}+\mathrm{H}_{2}$ where all the atoms are "light." The number of eigenvalues that it is necessary to include changes very slightly with temperature; at the highest temperature $(T=700 \mathrm{~K})$ about 24 Lanczos iterations are required. Note that the rate constant is within $2 \%$ of the final result after 14 iterations and within $1 \%$ after 18 iterations.

Results from helicity conserving calculations are presented in Figs. 5 and 6. The HCA rate constants for $K=0$ are plotted as a function of $J(J+1)$ on a semilog plot in Fig. 5

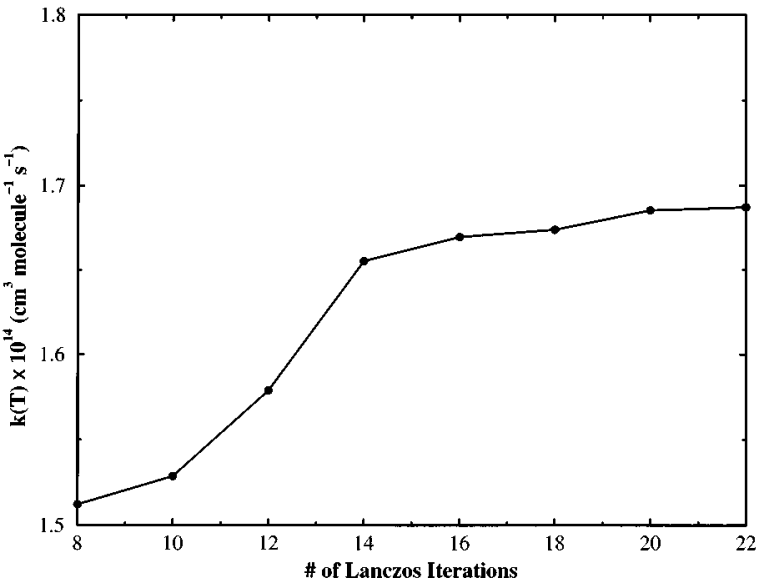

FIG. 4. The thermal rate constant vs the number of Lanczos iterations (i.e., the number of eigenvalues of the Boltzmannized flux operator used to calculate the trace) at $T=400 \mathrm{~K}$.

for $T=250$ and $400 \mathrm{~K}$. At both temperatures a straight line is obtained. Figure 6 shows the HCA rate constant as a function of $K^{2}$ for fixed $J$. Results are shown for $T=250 \mathrm{~K}$ with $J=24$ and $T=400 \mathrm{~K}$ with $J=24$ and 48. As shown on a semilog plot, $k_{J K}(T)$ vs $K^{2}$ is nonlinear but can be reasonably well approximated as a straight line. Note that for $T=400 \mathrm{~K}$, the slope of the line is independent of $J$.

These results can be interpreted in terms of the discussion in Section IIIB. In principle one needs to calculate $k_{J K}(T)$ within the HC approximation for all $J$ and $K$ that contribute. However, if the dependence of the rate on $J$ and $K$ is smooth, interpolation between the calculated values can be used to give the total rate constant. In that sense a rough "interpolation" can be made by assuming $\ln k_{J K}(T)$ $=-\alpha J(J+1)$ and $\ln k_{J K}(T)=-\eta K^{2}$ (i.e., fitting the curves as straight lines). This is equivalent to the $J$-shifting approximation (for fixed temperature) as discussed in Section III B and is tantamount to extracting "effective" moments of inertia. (We refer to rate constants obtained by this procedure

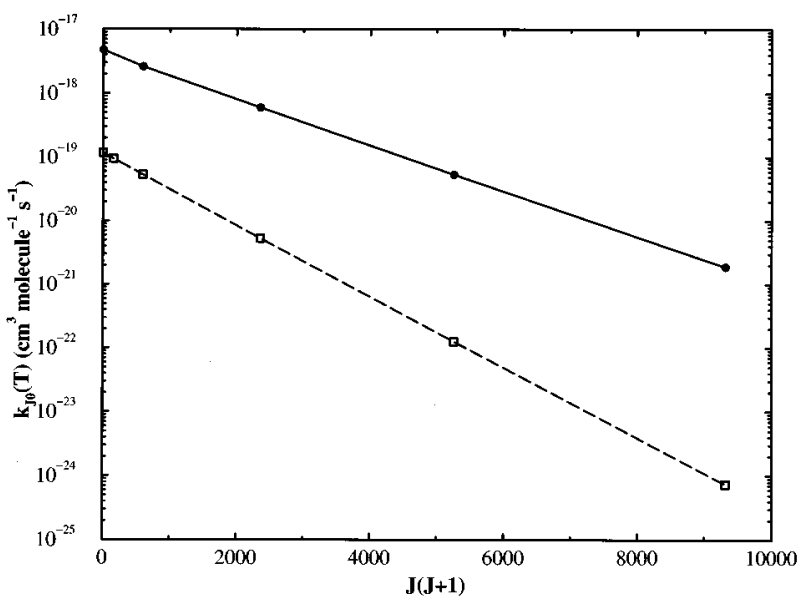

FIG. 5. The partial rate constant $k_{J K}$ (within the helicity conserving approximation) vs $J(J+1)$ for $K=0$. Results for $T=400 \mathrm{~K}$ (solid line with circles) and $T=250 \mathrm{~K}$ (dashed line with squares) are shown. 


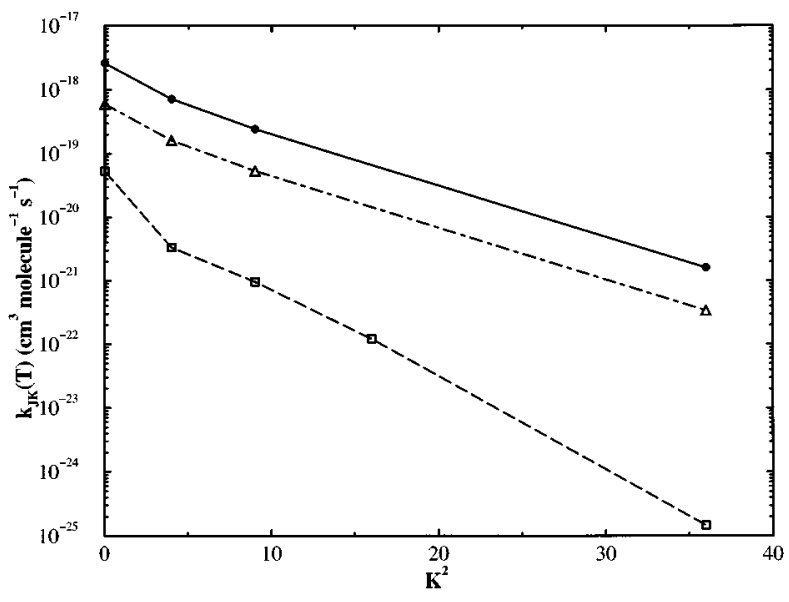

FIG. 6. The partial rate constant $k_{J K}$ (within the helicity conserving approximation) vs $K^{2}$ for $J$ fixed. Results are shown for $T=400 \mathrm{~K}$ with $J=24$ (solid line with circles) and $J=48$ (dot-dashed line with triangles) and $T=250 \mathrm{~K}$ with $J=24$ (dashed line with squares).

as HCA "adjusted.") Doing this we obtain at $I_{B}=4.73 \times 10^{5}$ a.u. and $I_{A}=1.96 \times 10^{3}$ a.u. at $T=400 \mathrm{~K}$ and $I_{B}=4.96 \times 10^{5}$ a.u. and $I_{A}=1.86 \times 10^{3}$ a.u. at $T=250 \mathrm{~K}$. This indicates that the moments of inertia do not depend strongly on temperature. In addition, these values are in reasonable agreement with those obtained by Koizumi et al. ${ }^{28}$ from the transition state geometry. The change in the value of the rate constant is $20 \%$ at $T=250 \mathrm{~K}$ and $8 \%$ at $T=400$ K. (See Table I and Fig. 7.)

Figure 7 presents an Arrhenius plot of the calculated rate constant as compared to the results of Koizumi et al. and experiment. ${ }^{37,41}$ The present results are larger than both the experimental and previous theoretical rates at all temperatures. Unfortunately, since the thermal rate constant is a highly averaged quantity, it is not possible to extract a particular feature of the potential energy surface to hold accountable for the discrepancy. With regard to experiment, if the barrier height were raised only $\sim 0.8-1.0 \mathrm{kcal} / \mathrm{mole}-$ recall that the $a b$ initio value of the barrier was scaled from 18.8 to $8.5 \mathrm{kcal} / \mathrm{mole}$ in the KSG potential energy surfacethen our calculated rates would be in much better agreement

TABLE I. Total thermal rate constants within the $J$-shifting and helicity conserving approximations compared for the three-dimensional $\mathrm{O}+\mathrm{HCl}$ reaction in units of $\mathrm{cm}^{3}$ molecule $\mathrm{e}^{-1} \mathrm{~s}^{-1}$.

\begin{tabular}{ccc}
\hline \hline & \multicolumn{2}{c}{$\mathrm{k}(\mathrm{T})$} \\
\cline { 2 - 3 } Temperature (K) & $J$-shifting & HCA “adjusted”, \\
\hline 200 & $9.8(-18)^{\mathrm{b}}$ & \\
250 & $1.0(-16)$ & $1.2(-16)$ \\
300 & $5.8(-16)$ & \\
350 & $2.0(-15)$ & \\
400 & $7.1(-15)$ & \\
500 & $3.7(-14)$ & \\
600 & $1.0(-13)$ & \\
700 & $2.8(-13)$ & \\
\hline \hline
\end{tabular}

${ }^{a}$ See Section IV.

${ }^{\mathrm{b}}$ The number in parentheses is the power of 10 .

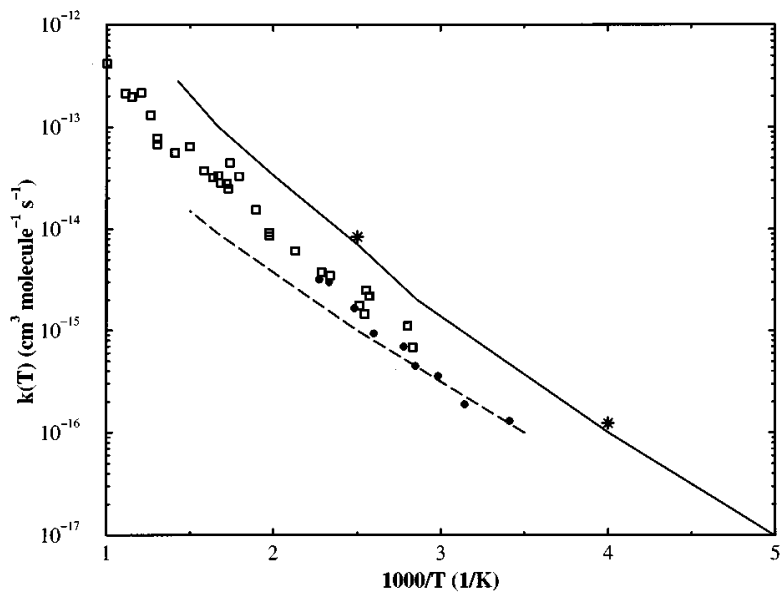

FIG. 7. Arrhenius plot of calculated and experimental thermal rate constants. The present $J$-shifted calculations are shown as a solid line and the results of Koizumi et al. as a dashed line. The asterisks are the present HCA "adjusted" rate constants (see the text). The experimental results of Brown and Smith (Ref. 37) are shown as filled circles and that of Mahamud et al. (Ref. 41) as open squares. (The results of Koizumi et al. were measured from their Fig. 2 and hence may be considered approximate.)

with experiment. The lack of agreement between our results and Koizumi et al.'s is harder to understand. At present we have no explanation for this. We note that the rate constants calculated by Moribayashi and Nakamura ${ }^{44}$ for the initial $\mathrm{HCl}$ rotational states $j=0,1,2$,- though not directly comparable to the present results since they are not fully Boltzmann averaged over all initial states-are also larger than the rates of Koizumi et al. (though smaller than ours). Moribayashi and Nakamura also suggest that higher $j$ states $(j>2)$ may contribute even more significantly to the rate and, if so, then their fully Boltzmann averaged rate constant would be even larger.

We note that in Fig. 7 the present results shown indicate that the slope of $\ln k(T)$ vs $1 / T$ increases with increasing temperature. This is also observed in the rates obtained by Koizumi et al. as well as in the experimental results shown here. In fact the activation energy reported by Brown and Smith over the temperature range $293-440 \mathrm{~K}$ is $5.9 \mathrm{kcal} / \mathrm{mole},{ }^{37}$ while a value of $7.3 \mathrm{kcal} / \mathrm{mole}$ is obtained by a least squares fit of the data of Mahamud, Kim, and Fontijn ${ }^{41}$ over the range 353-1486 K. Indeed, the results of Mahamud et al. show a non-Arrhenius increase in the activation energy as the temperature is raised. Our results give an activation energy of $5.7 \mathrm{kcal} / \mathrm{mole}$ over the range of $200-700 \mathrm{~K}$ as compared to about $5.0 \mathrm{kcal} / \mathrm{mole}$ from the results of Koizumi et al. over $285-667 \mathrm{~K}$. Thus the theoretically calculated activation energies are lower than those obtained by experiment though the correct non-Arrhenius behavior is reproduced.

We have also performed calculations for the thermal rate constant of the $\mathrm{O}+\mathrm{DCl}$ reaction. These are compared to the results for the $\mathrm{O}+\mathrm{HCl}$ reaction in Fig. 8. The deuterated rate constants were obtained by using the same basis set at a given temperature as was needed for the $\mathrm{O}+\mathrm{HCl}$ reaction. The most interesting result here is the tunneling enhance- 


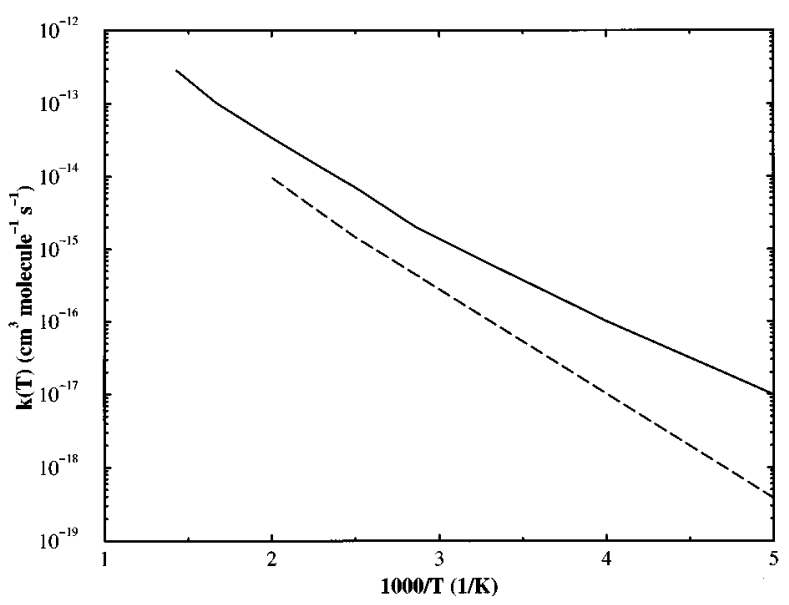

FIG. 8. Calculated thermal rate constants, $k(T)$ vs $1000 / T$ for the $\mathrm{O}+\mathrm{HCl}$ (solid line) and $\mathrm{O}+\mathrm{DCl}$ (dashed line) reactions.

ment of the rate when $\mathrm{H}$ is substituted for $\mathrm{D}$. While the thermal rate constants for the reaction with $\mathrm{DCl}$ follow an Arrhenius form (i.e., $\ln k(T)$ vs $1 / T$ is a straight line with the slope equal to the activation energy), with $\mathrm{HCl}$ the activation energy becomes smaller as the temperature is lowered. This change in the activation energy at lower temperatures can be attributed to the tunneling of the $\mathrm{H}$ atom. In the deuterated case the tunneling rate is reduced due to the heavier mass and the rate therefore remains Arrhenius at low temperature. Indeed, the activation energy for the $\mathrm{O}+\mathrm{DCl}$ reaction is 6.7 $\mathrm{kcal} / \mathrm{mole}$, larger than that for $\mathrm{O}+\mathrm{HCl}$. Experimental measurements of Brown and Smith found the ratio of the rate constants $k_{\mathrm{O}+\mathrm{HCl}} / k_{\mathrm{O}+\mathrm{DCl}}$ at $T=400 \mathrm{~K}$ as $2.4 \pm 0.7 .^{38}$ This is smaller by a factor of 2 than the ratio of 4.8 obtained in the present calculations.

\section{CONCLUDING REMARKS}

A method for directly calculating thermal rate constants for chemical reactions by means of the flux-flux autocorrelation function was presented. The method has three main features: (1) The low rank of the Boltzmannized flux operator is used to advantage in evaluating the quantum mechanical trace. An iterative Lanczos scheme is used to obtain the eigenfunctions of $\hat{F}(\beta)$ corresponding to the largest (in absolute value) eigenvalues and the trace is evaluated in this (much smaller) basis. (2) Absorbing potentials are used in the (real) time propagation to prevent reflection from the edge of the finite basis (here a DVR grid) making the method stable and thereby allowing the size of the basis to be reduced. (3) A split-operator algorithm is used for both the real and imaginary time propagation. For the real time propagation, this produces the time correlation function at all intermediate times necessary to perform the integral of $C_{f f}(t)$ at no additional computational effort.

We have applied this method to the calculation of thermal rate constants for the $\mathrm{O}+\mathrm{HCl}$ reaction over the temperature range $T=200-700 \mathrm{~K}$. Significant recrossing of the dividing surface is seen at all temperatures from the oscillations in the flux correlation function. It is found that about 20-24 Lanczos iterations are needed for the Boltzmannized flux operator to obtain the full rate constant, implying that, in this temperature range, between 9 and 12 states of the activated complex contribute significantly to the rate. The calculated rate constants display a non-Arrhenius temperature dependence in agreement with experiment. However, the present rate constants are larger than experimental results and previous theoretical calculations.

We have included the effects of nonzero total angular momentum using the $J$-shifting and helicity conserving approximations. These two approximations give results in reasonable agreement (within 10\%-20\%) with each other. It is expected that the HC approximation will be in good agreement with exact calculations since the projection quantum number along the $\mathrm{O}-\mathrm{Cl}$ axis should be well conserved.

Rate constants were also calculated for the $\mathrm{O}+\mathrm{DCl}$ reaction. In the deuterated reaction, the rates obey the Arrhenius relationship over the entire temperature range calculated ( $T=200-500 \mathrm{~K}$ ). Comparing these results to those for the $\mathrm{O}+\mathrm{HCl}$ reaction illustrates the effect of the tunneling enhancement of the rate constant at low temperatures. The reaction involving $\mathrm{H}$ shows a non-Arrhenius increase in the rate below about $350 \mathrm{~K}$ that is not present in the deuterated case.

\section{ACKNOWLEDGMENTS}

The authors would like to thank Dr. H. Koizumi and Professor George C. Schatz for providing them with the potential energy surface. They also wish to acknowledge Professor Schatz and Bill Poirer for many useful discussions. This work was supported by the Director, Office of Energy Research, Office of Basic Energy Sciences, Chemical Sciences Division of the U.S. Department of Energy under Contract No. DE-AC03-76SF00098. One author (W.H.T.) acknowledges support from the National Science Foundation.

\footnotetext{
${ }^{1}$ R. Jaquet and W. H. Miller, J. Phys. Chem. 89, 2139 (1985); K. Yamashita and W. H. Miller, J. Chem. Phys. 82, 5475 (1985); J. W. Tromp and W. H. Miller, J. Phys. Chem. 90, 3482 (1986); J. W. Tromp and W. H. Miller, Faraday Discuss. Chem. Soc. 84, 441 (1987); N. Makri and W. H. Miller, J. Chem. Phys. 90, 904 (1989); W. H. Miller, J. Phys. Chem. 99, 12387 (1995).

${ }^{2}$ W. H. Thompson and W. H. Miller, J. Chem. Phys. 102, 7409 (1995).

${ }^{3}$ U. Manthe, J. Chem. Phys. 102, 9205 (1995).

${ }^{4}$ (a) T. J. Park and J. C. Light, J. Chem. Phys. 85, 5870 (1986); (b) 88, 4897 (1988); (c) 91, 974 (1989); (d) 94, 2946 (1991); (e) 96, 8853 (1992).

${ }^{5}$ D. Brown and J. C. Light, J. Chem. Phys. 97, 5465 (1992).

${ }^{6}$ M. Thachuk and G. C. Schatz, J. Chem. Phys. 97, 7297 (1992); R. P. McRae, G. K. Schenter, B. C. Garrett, G. R. Haynes, G. A. Voth, and G. C. Schatz, ibid. 97, 7392 (1992); M. Thachuk, H. R. Mayne, and G. C. Schatz, ibid. 99, 3516 (1993); M. Thachuk and G. C. Schatz, ibid. 101, 6577 (1994)

${ }^{7}$ G. Wahnström and H. Metiu, Chem. Phys. Lett. 134, 531 (1987); G. Wahnström and H. Metiu, J. Phys. Chem. 92, 3240 (1988); G. Wahnström, B. Carmeli, and H. Metiu, J. Chem. Phys. 88, 2478 (1988).

${ }^{8}$ P. N. Day and D. G. Truhlar, J. Chem. Phys. 94, 2045 (1991).

${ }^{9}$ N. Rom, N. Moiseyev, and R. Lefebvre, J. Chem. Phys. 96, 8307 (1992); R. Lefebvre, V. Ryaboy, and N. Moiseyev, ibid. 98, 8601 (1993); V. Ryaboy and R. Lefebvre, ibid. 99, 9547 (1993).

${ }^{10}$ N. Makri, J. Chem. Phys. 94, 4949 (1991); M. Topaler and N. Makri, ibid. 101, 7500 (1994).
} 
${ }^{11}$ T. N. Truong, J. A. McCammon, D. J. Kouri, and D. K. Hoffman, J. Chem. Phys. 96, 8136 (1992).

${ }^{12}$ T. Yamamoto, J. Chem. Phys. 33, 281 (1960).

${ }^{13}$ W. H. Miller, S. D. Schwartz, and J. W. Tromp, J. Chem. Phys. 79, 4889 (1983).

${ }^{14}$ W. H. Miller, J. Chem. Phys. 61, 1823 (1974).

${ }^{15}$ D. O. Harris, G. G. Engerholm, and W. D. Gwinn, J. Chem. Phys. 43, 1515 (1965).

${ }^{16}$ J. V. Lill, G. A. Parker, and J. C. Light, Chem. Phys. Lett. 89, 483 (1982); J. C. Light, I. P. Hamilton, and J. V. Lill, J. Chem. Phys. 82, 1400 (1985); Z. Bačić and J. C. Light, ibid. 85, 4594 (1986); R. M. Whitnell and J. C. Light, ibid. 89, 3674 (1988).

${ }^{17}$ D. T. Colbert and W. H. Miller, J. Chem. Phys. 96, 1982 (1992).

${ }^{18}$ A. Goldberg and B. W. Shore, J. Phys. B 11, 3339 (1978).

${ }^{19}$ C. Leforestier and R. E. Wyatt, J. Chem. Phys. 78, 2334 (1983).

${ }^{20}$ R. Kosloff and D. Kosloff, J. Comput. Phys. 63, 363 (1986).

${ }^{21}$ D. Neuhauser and M. Baer, J. Chem. Phys. 90, 4351 (1989); D. Neuhauser and M. Baer, ibid. 91, 4651 (1989); D. Neuhauser, M. Baer, and D. J. Kouri, ibid. 93, 2499 (1990).

${ }^{22}$ G. Jolicard and E. J. Austin, Chem. Phys. Lett. 121, 106 (1985); G. Jolicard and E. J. Austin, ibid 103, 295 (1986); G. Jolicard and M. Y. Perrin, ibid. 116, 1 (1987); G. Jolicard, C. Leforestier, and E. J. Austin, J. Chem. Phys. 88, 1026 (1988).

${ }^{23}$ I. Last, D. Neuhauser, and M. Baer, J. Chem. Phys. 96, 2017 (1992); I. Last and M. Baer, Chem. Phys. Lett. 189, 84 (1992); I. Last, A. Baram, and M. Baer, ibid. 195, 435 (1992); I. Last, A. Baram, H. Szichman, and M. Baer, J. Phys. Chem. 97, 7040 (1993).

${ }^{24}$ T. Seideman and W. H. Miller, J. Chem. Phys. 96, 4412 (1992); 97, 2499 (1992); W. H. Miller and T. Seideman, in Time Dependent Quantum Molecular Dynamics: Experiment and Theory, edited by J. Broeckhove (NATO, ARW, 1992).
${ }^{25}$ T. Seideman and W. H. Miller, J. Chem. Phys. 95, 1768 (1991).

${ }^{26}$ C. Lanczos, J. Res. Natl. Bur. Stand. 45, 255 (1950).

${ }^{27}$ J. M. Bowman, J. Phys. Chem. 95, 4960 (1991).

${ }^{28}$ H. Koizumi, G. C. Schatz, and M. S. Gordon, J. Chem. Phys. 95, 6421 (1991).

${ }^{29}$ R. T Pack, J. Chem. Phys. 60, 633 (1974).

${ }^{30}$ P. McGuire and D. J. Kouri, J. Chem. Phys. 60, 2488 (1974).

${ }^{31}$ J. Tennyson and J. R. Henderson, J. Chem. Phys. 91, 3815 (1989).

${ }^{32}$ S. E. Choi and J. C. Light, J. Chem. Phys. 92, 2129 (1990).

${ }^{33}$ M. Mladenović and Z. Bacić, J. Chem. Phys. 93, 3039 (1990).

${ }^{34}$ C. Leforestier, J. Chem. Phys. 94, 6388 (1991).

${ }^{35}$ See, for example, D. H. Zhang and J. Z. H. Zhang, J. Chem. Phys. 101, 1146 (1994).

${ }^{36}$ M. S. Gordon, K. K. Baldridge, R. J. Bartlett, and D. Bernhold, Chem. Phys. Lett. 158, 189 (1989).

${ }^{37}$ R. D. H. Brown and I. W. M. Smith, Int. J. Chem. Kinet. 7, 301 (1975).

${ }^{38}$ R. D. H. Brown and I. W. M. Smith, Int. J. Chem. Kinet. 10, 1 (1978).

${ }^{39}$ D. L. Singleton and R. J. Cvetanovic, Int. J. Chem. Kinet. 13945 (1981).

${ }^{40}$ I. W. M. Smith in Bimolecular Collisions edited by by M. N. R. Ashfold and J. E. Baggott (Royal Society of Chemistry, 1989), p. 53.

${ }^{41}$ K. Mahamud, J.-S. Kim, and A. Fontijn, J. Phys. Chem. 94, 2994 (1990).

${ }^{42}$ D. J. Rakestraw, K. G. McKendrick, and R. N. Zare, J. Chem. Phys. 87, 7341 (1987); R. Zhang, W. J. van der Zande, M. J. Bronikowski, and R. N. Zare, ibid. 94, 2704 (1991).

${ }^{43}$ A. Persky and M. Broida, J. Chem. Phys. 81, 4352 (1984).

${ }^{44}$ K. Moribayashi and H. Nakamura, J. Phys. Chem. 99, 15410 (1995).

${ }^{45}$ B. Ramachandran, J. Senekowitsch, and R. E. Wyatt, J. Mol. Struct. (Theochem) (to be published).

${ }^{46} \mathrm{~B}$. Ramachandran (private communication). 